\title{
Electron bunching in triple quantum dot interferometers
}

\author{
Fernando Domínguez, Gloria Platero, Sigmund Kohler \\ Instituto de Ciencia de Materiales de Madrid, CSIC, Cantoblanco, E-28049 Madrid, Spain
}

\begin{abstract}
We study electron transport through a triple quantum dot in ring or interferometer configuration. In particular, we analyze the influence of a gate voltage that detunes one of the dots, such that it becomes off-resonant. In this regime, interference effects fade away, i.e., the current becomes independent of a penetrating flux. Despite the absence of interference effects, the off-resonant dot causes intriguing noise properties which we characterize by the full-counting statistics of the transported electrons. It turns out that the detuning causes strong electron bunching. Analytical results for limiting cases support this picture. A possible application is the construction of current sources with widely tunable noise properties.
\end{abstract}

Keywords: quantum dots, quantum transport, full counting statistics

PACS: 73.23.-b, 05.60.Gg, 74.50.+r

\section{Introduction}

During the last decade, a huge effort has been made to understand the conduction properties of quantum systems that consist of only a few discrete levels. Spurred by Feyn'man's vision of "plenty of room at the bottom" [1], it has e.g. been proposed to use single molecules as elements of future electronic circuits [2]. Although this task is far from being accomplished, molecular electronics already became an established field [3, 4]. Apart from their technological promises, conducting molecules may also serve as tools for the implementation of fundamental physical phenomena. One example is the intriguing effect of tunnel suppression by the purely coherent influence of an ac field [5], which is stable even in the presence of Coulomb repulsion [6]. Coherent destruction of tunneling leaves its fingerprints in the transport characteristics of laser-driven molecular 'wires, where an ac field may suppress the current and its fluctuations [7, 8, 9, 10].

From a theoretical perspective, molecular wires share many features with quantum dots. In particular, the electronic structure of both systems consists of discrete states. 'Owing to this fact, coherently coupled quantum dots may be considered as "artificial molecules", despite the fact that their energy scales are several orders of magnitude smaller than those of real molecules. For roughly one decade, the state of the art has been to couple just two quantum dots coherently [11, 12, 13], while triple quantum dots have been realized only recently [14, 15, 16. Both double quantum dots [17] and triple quantum dots [15, 16] can be constructed such that electrons coming from the source may proceed on two different paths towards the drain. There they interfere constructively or destructively, depending on the setup and a possible flux enclosed by the interfering paths. Moreover, there exist dark states which are superpositions of states that are decoupled from the drain and, thus, block the electron transport [18, 19]. Such blockade may be resolved by excitations with proper ac fields [20, 21] which also create spin correlations between transported electrons 22].

Here we investigate the conduction properties of a triple quantum dot in ring configuration as sketched in Fig. 1. A penetrating flux allows the operation as Aharonov-Bohm interferometer. We focus on the influence of a gate voltage that allows shifting of the levels of dot 2 out of resonance. The intuitive expectation is that for strong detuning, the two other dots govern the transport process, such that the triple quantum dot behaves like a double quantum dot. While this is indeed the case for the current, electrons temporarily trapped in dot 2 may cause Coulomb blockade and, thus, interrupt the transport such that the electron flow becomes avalanche-like. This is reflected in the zero-frequency component of the current-current correlation function which significantly exceeds the value for a Poisson process. With this criterion, avalanche-like transport has been predicted for quantum dots coupled to a harmonic mode [23, 24], multi-level quantum dots 25], triple quantum dots in the Kondo regime [26], and also when destructive interference effects strongly suppress the current [27, 28, 29, 30].

An established theoretical tool for characterizing such correlated transport is full counting statistics [25, 31, 32 which provides the complete information about the distribution of the transported charge in terms of the corresponding cumulants. For a Markovian master equation as employed below, it is possible to express these cumulants as derivatives of a particular eigenvalue of the Liouville 


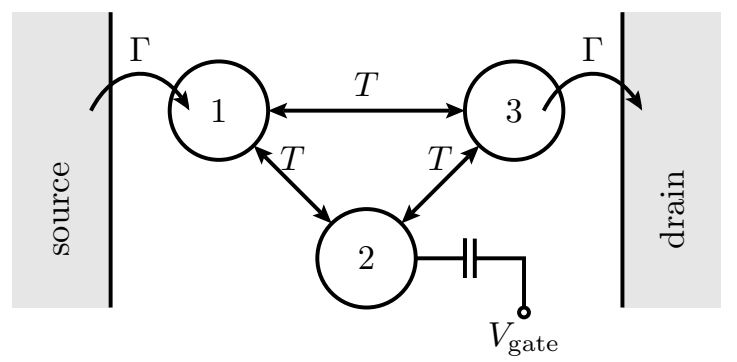

Figure 1: Triple quantum dot in ring configuration coupled to electron source and drain. The onsite energies of dots 1 and 3 are $\epsilon_{1,3}=0$, while the onsite energy of dot 2 can be tuned by a gate voltage such that $\epsilon_{2}=e V_{\text {gate }}$. The bias voltage $V$ is assumed to shift the chemical potentials symmetrically, such that $\mu_{\mathrm{L}}=\mathrm{eV} / 2=-\mu_{\mathrm{R}}$

operator augmented by a counting variable [32]. Generally, this requires the computation of derivatives of high order. As soon as one has to rely on a numerical treatment, practical calculations may represent a formidable task. Recently, Flindt et al. have found a way to circumvent this difficulty [33, 34]. Based on Rayleigh-Schrödinger perturbation theory, they derived a scheme that allows one to recursively compute the full counting statistics. We use this approach for our numerical solution.

Our paper is organized as follows. In Section 2 we introduce a system-lead Hamiltonian and derive a master equation formalism for the computation of the full counting statistics. The numerical results for the current and the zero-frequency noise, presented in Section 3 , provide information about the super-Poissonian nature of the transport process. In Section 4, we derive for the cumulants up to fourth order analytical expressions valid in the low-bias regime.

\section{Model and master equation approach}

\subsection{Dot-lead Hamiltonian}

We consider a triple quantum dot with a ring-shaped geometry as depicted in Fig. 1, which is described by the Hamiltonian

$$
H_{\mathrm{TQD}}=\sum_{i} \epsilon_{i} c_{i}^{\dagger} c_{i}+T \sum_{i, j<i}\left(c_{i}^{\dagger} c_{j}+c_{j} c_{i}^{\dagger}\right)+U \sum_{i, j<i} c_{i}^{\dagger} c_{i} c_{j}^{\dagger} c_{j},
$$

where $i, j=1,2,3$ label the dots, while $c_{i}^{\dagger}$ and $c_{i}$ denote the corresponding creation and annihilation operator of a spinless electron. The first term refers to the onsite energy $\epsilon_{i}$ of an electron on dot $i$, for which we assume that $\epsilon_{1}=\epsilon_{3}=0$, while $\epsilon_{2}=e V_{\text {gate }}$ can be tuned by a gate voltage. The second term describes electron tunneling between the dots, for which we assume that the tunnel matrix element $T$ is the same for all three possible transitions. The last contribution models inter-dot Coulomb repulsion with strength $U$. In the present case, this term can be written in the form $\frac{1}{2} U N(N-1)$, where $N$ denotes the total number of electrons on the three dots. We assume that the intra-dot interaction is practically infinite, which corresponds to modeling each dot by a single level.

In a ring-shaped geometry, the electrons possess two paths for traveling from the source to the drain. Thus, interference plays a role. This interference can be controlled by a magnetic flux $\Phi$ penetrating the ring. We capture this by the replacement $c_{1}^{\dagger} c_{3} \rightarrow c_{1}^{\dagger} c_{3} \exp \left(2 \pi \Phi / \Phi_{0}\right)$, where $\Phi_{0}=h / e$ denotes the flux quantum. Note that since we consider only spinless electrons, the effect of an associated Zeeman splitting is ignored. Nevertheless, the flux dependence of the current provides information about the relevance of interference effects.

Dots 1 and 3 are attached to electron reservoirs with chemical potentials $\mu_{\mathrm{L}}$ and $\mu_{\mathrm{R}}$ such that the bias voltage fulfills $e V=\mu_{\mathrm{L}}-\mu_{\mathrm{R}}$. They are described by the lead Hamiltonian

$$
H_{\text {leads }}=\sum_{\ell, q} \epsilon_{q} c_{\ell q}^{\dagger} c_{\ell q}
$$

where $\ell=\mathrm{L}, \mathrm{R}$, and the expectation value $\left\langle c_{\ell q}^{\dagger} c_{\ell^{\prime} q^{\prime}}\right\rangle=$ $f\left(\epsilon_{q}-\mu_{\ell}\right) \delta_{\ell \ell^{\prime}} \delta_{q q^{\prime}} \equiv f_{\ell}\left(\epsilon_{q}\right) \delta_{\ell \ell^{\prime}} \delta_{q q^{\prime}}$ with chemical potential $\mu_{\ell}$ and the Fermi function $f(x)=\left[\exp \left(x / k_{B} T\right)+1\right]^{-1}$. The dot-lead contact is established by the tunnel Hamiltonian

$$
H_{\mathrm{tun}}=\sum_{q}\left(V_{\mathrm{L} q} c_{\mathrm{L} q}^{\dagger} c_{1}+V_{\mathrm{R} q} c_{\mathrm{R} q}^{\dagger} c_{3}\right)+\text { h.c. }
$$

We assume within a wide-band limit that all effective coupling strengths $\Gamma_{\ell}(\epsilon)=2 \pi \sum_{q}\left|V_{\ell q}\right|^{2} \delta\left(\epsilon-\epsilon_{q}\right)$ are energy independent and that the setup is symmetric such that $\Gamma_{\mathrm{L}}=\Gamma_{\mathrm{R}}=\Gamma$.

\subsection{Markovian master equation}

The derivation of a master equation starts from the Liouville-von Neumann equation for the full density operator, $\mathrm{i} \hbar \dot{R}=\left[H_{\mathrm{TQD}}+H_{\text {tun }}+H_{\text {leads }}, R\right]$. By standard techniques [35], one obtains for the reduced density operator of the central system, $\rho=\operatorname{tr}_{\text {leads }} R$, within second-order perturbation theory the Bloch-Redfield equation

$$
\begin{aligned}
\dot{\rho} & =-\frac{\mathrm{i}}{\hbar}\left[H_{\mathrm{TQD}}, \rho\right]-\frac{1}{\hbar^{2}} \operatorname{tr}_{\text {leads }} \int_{0}^{\infty} \mathrm{d} \tau\left[H_{\mathrm{tun}},\left[\tilde{H}_{\mathrm{tun}}(-\tau), R\right]\right] \\
& \equiv \mathcal{L} \rho
\end{aligned}
$$

which can be evaluated under the factorization assumption $R=\rho_{\text {leads }, 0} \otimes \rho$. The tilde denotes the interaction picture with respect to the uncoupled Hamiltonian, $\tilde{X}(t)=$ $U_{0}^{\dagger}(t) X U_{0}(t)$, where $U_{0}(t)=\exp \left\{-\mathrm{i}\left(H_{\mathrm{TQD}}+H_{\text {leads }}\right) t / \hbar\right\}$. We proceed by inserting the dot-lead tunnel Hamiltonian (3) and evaluate the trace over the lead states. In order to cope with the interaction picture, we decompose the resulting master equation into the eigenstates $|\alpha\rangle$ of $H_{\mathrm{TQD}}$, where $E_{\alpha}$ denotes the corresponding eigenenergy. This allows us to evaluate the $\tau$-integration, which yields a delta function and a principal value term. Neglecting the latter, 
we obtain for the density matrix elements the equation of motion

$$
\dot{\rho}_{\alpha \beta}=-\frac{\mathrm{i}}{\hbar}\left(E_{\alpha}-E_{\beta}\right) \rho_{\alpha \beta}+\sum_{\alpha^{\prime}, \beta^{\prime}} \mathcal{L}_{\alpha \beta, \alpha^{\prime} \beta^{\prime}} \rho_{\alpha^{\prime} \beta^{\prime}}
$$

with the incoherent dot-lead tunneling given by

$$
\begin{aligned}
& \mathcal{L}_{\alpha \beta, \alpha^{\prime} \beta^{\prime}} \\
& =\sum_{\ell=1,3} \frac{\Gamma_{\ell}}{2}\left\{\left\langle\alpha\left|c_{\ell}^{\dagger}\right| \alpha^{\prime}\right\rangle\left\langle\beta^{\prime}\left|c_{\ell}\right| \beta\right\rangle f_{\ell}\left(E_{\alpha}-E_{\alpha^{\prime}}\right)\right. \\
& +\left\langle\alpha\left|c_{\ell}^{\dagger}\right| \alpha^{\prime}\right\rangle\left\langle\beta^{\prime}\left|c_{\ell}\right| \beta\right\rangle f_{\ell}\left(E_{\beta}-E_{\beta^{\prime}}\right) \\
& -\sum_{\gamma}\left\langle\beta^{\prime}\left|c_{\ell}\right| \gamma\right\rangle\left\langle\gamma\left|c_{\ell}^{\dagger}\right| \beta\right\rangle f_{\ell}\left(E_{\gamma}-E_{\beta^{\prime}}\right) \delta_{\alpha \alpha^{\prime}} \\
& \left.-\sum_{\gamma}\left\langle\alpha\left|c_{\ell}\right| \gamma\right\rangle\left\langle\gamma\left|c_{\ell}^{\dagger}\right| \alpha^{\prime}\right\rangle f_{\ell}\left(E_{\gamma}-E_{\alpha^{\prime}}\right) \delta_{\beta \beta^{\prime}}\right\} \\
& +\left(c_{\ell}, c_{\ell}^{\dagger}, f_{\ell}\right) \rightarrow\left(c_{\ell}^{\dagger}, c_{\ell}, 1-f_{\ell}\right)
\end{aligned}
$$

where the leads now are labeled by the number $\ell$ of the dot to which they are attached. Electron tunneling from the leads to the dots is described by the explicitly written terms, while the replacement in the last line yields the terms for tunneling from the dots to the leads.

The solution of the master equation (5) contains the full information about the state of the central system and provides all corresponding expectation values. However, we are interested in the statistics of the charge transported after the initial preparation, which is an expectation value of lead operators. Therefore we have to generalize the master equation formalism by introducing a counting variable which allows one to keep track of this information.

\subsection{Full-counting statistics}

The counting variable $\chi$ is defined via the moment generating function

$$
\phi(\chi, t)=\left\langle\exp \left(\mathrm{i} \chi N_{\mathrm{R}}\right)\right\rangle_{t}
$$

where $N_{\mathrm{R}}=\sum_{q} c_{3 q}^{\dagger} c_{3 q}$ is the electron number operator of the right lead, while the angular brackets refer to the expectation value at time $t$. The $k$ th derivative of $\phi(\chi, t)$ with respect to i $\chi$ at $\chi=0$ obviously is the moment $\left\langle N_{\mathrm{R}}^{k}\right\rangle$ of the electron distribution in the right lead. The cumulants of the distribution are defined as the corresponding derivatives of $\ln \phi(\chi, t)$. For a Markov process, they eventually become linear in time. Their time-derivatives at large times,

$$
C_{k}=\left.\frac{\partial}{\partial t} \frac{\partial^{k}}{\partial(\mathrm{i} \chi)^{k}} \ln \phi(\chi, t)\right|_{\chi=0, t \rightarrow \infty}
$$

are the stationary current cumulants and characterize the transport. The first and the second cumulant, $C_{1}$ and $C_{2}$, are essentially the current $I=e C_{1}$ and its zero-frequency noise $S=e^{2} C_{2}$, respectively. Their ratio, the Fano factor $F=C_{2} / C_{1}$, represents a dimensionless measure for the noise of the transport process [36]. It is defined such that for a Poissonian process $F=1$.

Our goal is now to find a reduced master equation that allows one to compute $\phi(\chi, t)$. We start again from the full density operator $R$, but now multiply it with the operator $\exp \left(\mathrm{i} \chi N_{\mathrm{R}}\right)$ before tracing out the leads. This yields the generalized reduced density operator $P(\chi, t)=$ $\operatorname{tr}_{\text {leads }}\left\{\exp \left(\mathrm{i} \chi N_{\mathrm{R}}\right) R\right\}$ which contains information about the electron distribution of the drain and fulfills the trace condition $\operatorname{tr} P=\phi$. Using the commutation relations $\left[N_{\mathrm{R}}, \Lambda\right]=\Lambda$ and $\left[N_{\mathrm{R}}, \Lambda^{\dagger}\right]=-\Lambda^{\dagger}$, where $\Lambda=\sum_{q} V_{q} c_{3}^{\dagger} c_{3 q}$ we obtain the master equation

$$
\dot{P}(\chi, t)=\mathcal{L}_{\chi} P(\chi, t)
$$

with the augmented Liouvillian

$$
\mathcal{L}_{\chi}=\mathcal{L}+\left(\mathrm{e}^{\mathrm{i} \chi}-1\right) \mathcal{J}^{+}+\left(\mathrm{e}^{-\mathrm{i} \chi}-1\right) \mathcal{J}^{-}
$$

and the particle current superoperators $\mathcal{J}^{ \pm}$. After some algebra, we find for the latter the expression [37]

$$
\mathcal{J}^{-} \rho=\frac{e \Gamma_{\ell}}{2 \pi} \int_{0}^{\infty} \mathrm{d} \tau \int \mathrm{d} \epsilon \mathrm{e}^{-\mathrm{i} \epsilon \tau} \tilde{c}_{3}^{\dagger}(-\tau) \rho c_{3} f_{\ell}(\epsilon)+\text { h.c. }
$$

and $\mathcal{J}^{+}$is formally obtained from $\mathcal{J}^{-}$by the replacement $\left(c_{3}^{\dagger}, c_{3}, f_{\mathrm{R}}\right) \rightarrow\left(c_{3}, c_{3}^{\dagger}, 1-f_{\mathrm{R}}\right)$. The superoperator $\mathcal{J}^{+}$describes tunneling from dot 3 to the right lead, while $\mathcal{J}^{-}$ corresponds to the reversed process. We proceed as for the derivation of the master equation (5) and decompose the current superoperators into the eigenstates of $H_{\mathrm{TQD}}$ which yields

$$
\begin{aligned}
\mathcal{J}_{\alpha \beta, \alpha^{\prime} \beta^{\prime}}^{+}= & \frac{\Gamma_{\mathrm{R}}}{2}\left\langle\alpha\left|c_{3}\right| \alpha^{\prime}\right\rangle\left\langle\alpha^{\prime}\left|c_{3}^{\dagger}\right| \beta\right\rangle \\
& \times\left\{2-f_{\mathrm{R}}\left(E_{\alpha}-E_{\alpha^{\prime}}\right)-f_{\mathrm{R}}\left(E_{\beta}-E_{\beta^{\prime}}\right)\right\}, \\
\mathcal{J}_{\alpha \beta, \alpha^{\prime} \beta^{\prime}}^{-}= & \frac{\Gamma_{\mathrm{R}}}{2}\left\langle\alpha\left|c_{3}^{\dagger}\right| \alpha^{\prime}\right\rangle\left\langle\alpha^{\prime}\left|c_{3}\right| \beta\right\rangle \\
& \times\left\{f_{\mathrm{R}}\left(E_{\alpha}-E_{\alpha^{\prime}}\right)+f_{\mathrm{R}}\left(E_{\beta}-E_{\beta^{\prime}}\right)\right\} .
\end{aligned}
$$

In the long-time limit, the dynamics of $P(\chi, t)$ is governed by the eigenvalue of $\mathcal{L}_{\chi}$ with the largest real part, denoted as $\lambda(\chi)$. We assume that $\lambda(\chi)$ can be uniquely identified by its $\operatorname{limit}_{\chi \rightarrow 0} \lambda(\chi)=0$, i.e., it corresponds to the stationary solution of the Liouvillian $\mathcal{L}=\mathcal{L}_{\chi \rightarrow 0}$. Then, $P=A(\chi) \exp [\lambda(\chi) t]$, with $A(\chi)$ be the corresponding "eigenoperator" of $\mathcal{L}_{\chi}$. It is straightforward to see that $\ln \phi(\chi, t)=\ln \operatorname{tr} A(\chi)+\lambda(\chi) t$. In the long-time limit, the contribution of the normalization factor is not relevant and, thus, we can conclude that $\lambda(\chi)$ is the current cumulant generating function 32]. It can be written as the series

$$
\lambda(\chi)=\sum_{k=1}^{\infty} \frac{C_{k}}{k !}(\mathrm{i} \chi)^{k} .
$$

The remaining task is now to compute the proper eigenvalue of $\mathcal{L}_{\chi}$ and its derivatives with respect to $\chi$. In many cases, the reduced density matrix has a sufficiently small 
dimension or possesses symmetries, such that one can continue with analytical calculations. As soon as one has to resort to a numerical treatment, however, one faces the difficulty of numerically computing derivatives. This can be avoided with the recursive scheme developed in Ref. [33] even for non-Markovian master equations. Here we restrict ourselves to the Markovian limit.

Since we are interested in the derivatives of the cumulant generating function at $\chi=0$, we can treat $\chi$ as small parameter and employ perturbation theory. Then the series (14) for the eigenvalue $\lambda(\chi)$ corresponds to the usual ansatz for the eigenvalue. It can be computed by the recursion derived in Appendix Appendix A see Eqs. A.6) and (A.7). Upon setting in these expressions $E_{k}=C_{k} / k$ !, $V_{k}=\left[\mathcal{J}^{+}+(-1)^{k} \mathcal{J}^{-}\right] / k$ !, and $P_{k}=\left|\phi_{k}\right\rangle / k$ !, we find

$$
\begin{aligned}
C_{k} & =\sum_{k^{\prime}=0}^{k-1}\left(\begin{array}{c}
k \\
k^{\prime}
\end{array}\right) \operatorname{tr}\left\{\mathcal{J}^{+}+(-1)^{k-k^{\prime}} \mathcal{J}^{-}\right\} P_{k^{\prime}} \\
P_{k} & =\frac{\mathcal{Q}}{\mathcal{L}} \sum_{k^{\prime}=0}^{k-1}\left(\begin{array}{c}
k \\
k^{\prime}
\end{array}\right)\left\{C_{k-k^{\prime}}-\left[\mathcal{J}^{+}+(-1)^{k-k^{\prime}} \mathcal{J}^{-}\right]\right\} P_{k^{\prime}}
\end{aligned}
$$

The iteration starts from the stationary solution of the Liouvillian, $P_{0}=\left|\phi_{0}\right\rangle=\rho_{\infty}$, which implies $E_{0}=C_{0}=0$. Multiplication with the corresponding left eigenvector $\left\langle\phi_{0}\right|$ must correspond to computing the trace. This becomes clear when one notes that the Liouvillian is trace conserving, i.e. $\operatorname{tr} \mathcal{L} X=0$ for any operator $X$. Consequently, $\mathcal{Q}=\left(\mathbf{1}-\rho_{\infty} \operatorname{tr}\right)$ is the projector on the subspace perpendicular to $\rho_{\infty}$. In this subspace, the Liouvillian possesses the pseudo-inverse $\mathcal{Q} / \mathcal{L}$. Applying it to any operator, i.e. computing $(\mathcal{Q} / \mathcal{L}) B \equiv X$, is equivalent to solving the linear equation $\mathcal{L} X=B$ under the constraint $\operatorname{tr} X=0$.

In particular, we obtain for the first two cumulants, which are the current and the zero-frequency noise, the known expressions [23]

$$
\begin{aligned}
I= & e \operatorname{tr}\left(\mathcal{J}^{+}-\mathcal{J}^{-}\right) \rho_{\infty} \\
S= & e^{2} \operatorname{tr}\left(\mathcal{J}^{+}+\mathcal{J}^{-}\right) \rho_{\infty} \\
& \quad-2 e^{2} \operatorname{tr}\left(\mathcal{J}^{+}-\mathcal{J}^{-}\right) \frac{\mathcal{Q}}{\mathcal{L}}\left(\mathcal{J}^{+}-\mathcal{J}^{-}\right) \rho_{\infty}
\end{aligned}
$$

\section{Numerical observations}

An intuitive picture for the transport properties as a function of the gate voltage can be provided for the limiting cases in which the detuning of dot 2 is either much larger or much smaller than the inter-dot tunneling: In the limit $\left|e V_{\text {gate }}\right| \ll T$, all three dots are in resonance and, thus, the electrons may take with similar probability two different routes, namely $|1\rangle \rightarrow|3\rangle$ and $|1\rangle \rightarrow|2\rangle \rightarrow|3\rangle$. Therefore one expects interference to be relevant, such that the current can be modified by a magnetic flux penetrating the ring. If the gate voltage is large, by contrast, i.e. for $\left|e V_{\text {gate }}\right| \gg T$, dot 2 is off-resonant and, thus, should be

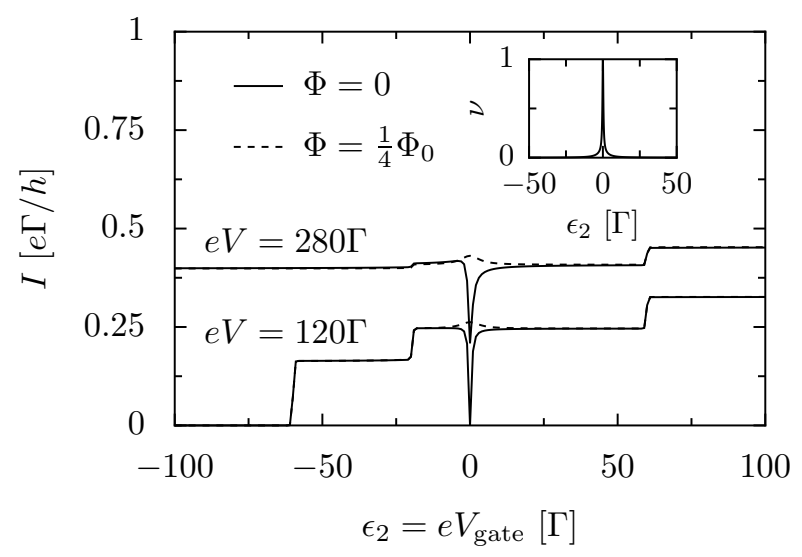

Figure 2: Current as a function of the gate voltage $V_{\text {gate }}=\epsilon_{2} / e$ for different magnetic fluxes $\Phi$ and bias voltages. The inter-dot tunnel coupling and interaction are $T=2 \Gamma$ and $U=80 \Gamma$, respectively. Inset: Corresponding visibility for $e V=120 \Gamma$.

of minor relevance. Therefore, the transport properties of the setup are expected to be essentially those of a double quantum dot formed by dots 1 and 3. Our numerical results, however, will demonstrate that this is only true for the average current, while the current noise is significantly altered by the presence of dot 2 even when far detuned.

As a first step, we solve the master equation numerically. In doing so, we identify parameter regimes in which the stationary state of the triple dot is dominated by a few eigenstates. This allows us to reduce the complexity of the master equation, such that we can achieve an analytical treatment that provides insight to the transport mechanism. For the numerical solution, we use the dot-lead coupling $\Gamma$ as energy unit. For the typical value $\Gamma=10 \mu \mathrm{eV}$, the corresponding current unit reads $e \Gamma / \hbar=2.43 \mathrm{nA}$.

\subsection{Stationary current and occupation probabilities}

The stationary current as a function of the gate voltage $V_{\text {gate }}=\epsilon_{2} / e$ is shown in Fig. 2. It demonstrates that for $\left|\epsilon_{2}\right| \lesssim T$, the current indeed is significantly flux dependent as conjectured above. For flux $\Phi=0$, the current assumes its minimum. This can be explained by the fact that for $\Phi=0=\epsilon_{2}$, the state $\left|\psi_{\text {dark }}\right\rangle=(|1\rangle-|2\rangle) / \sqrt{2}$ is an eigenstate of the triple dot Hamiltonian and is orthogonal to state $|3\rangle$. Therefore it is not directly coupled to the right lead, which has the consequence that $\left|\psi_{\text {dark }}\right\rangle$ may trap an electron which henceforth blocks transport 38. This is formally related to an atomic state that is decoupled from a light field and, thus, remains invisible [39].

As a criterion for the relevance of interference, we compute the visibility

$$
\nu=\frac{I_{\max }-I_{\min }}{I_{\max }+I_{\min }}
$$

where $I_{\max }=\max _{\Phi} I(\Phi)$ is the maximal current upon flux variation for a given gate voltage, and $I_{\min }$ is defined accordingly. The inset of Fig. 2 demonstrates that for 


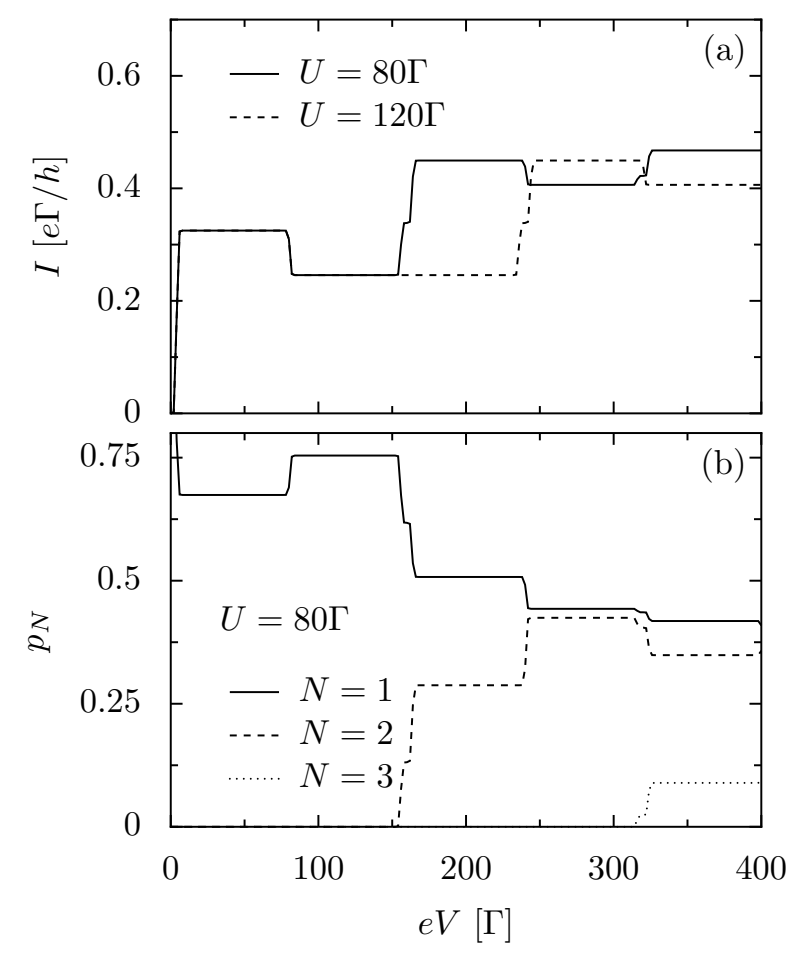

Figure 3: (a) Current as a function of the bias voltage $e V=\mu_{\mathrm{L}}-\mu_{\mathrm{R}}$ for various interaction strengths. The inter-dot tunnel coupling and the gate voltage are $T=2 \Gamma$ and $V_{\text {gate }}=\epsilon_{2} / e=40 \Gamma / e$, respectively. (b) Probability that for $U=80 \Gamma$, the asymptotic state of the triple quantum dot contains $N$ electrons.

$\left|\epsilon_{2}\right| \gg T$, interference plays a minor role. The reason for this is that when dot 2 is strongly detuned, transport through this off-resonant dot requires co-tunneling and, thus, the path via dot 2 has a significantly lower probability than the direct path.

Henceforth we focus on the region without interference effects. There a main feature of the current is that it exhibits plateaus, see Fig. 3(a). This is consistent with the usual Coulomb blockade scenario in which the bias and the gate voltage, determine the maximal number of electrons that can reside on the dots. Accordingly, the probability for having a particular dot occupation changes at the steps, as can be appreciated from Fig. 3(b). Moreover, an electron can tunnel from a left lead to the dots only if it has sufficient energy to compensate the Coulomb repulsion of the electrons that are already in the triple quantum dot. Thus the occupation with $N$ electrons $(N=1,2,3)$ is possible only if one chemical potential is larger than $(N-1) U$. For the symmetric positive voltage drop assumed herein, the larger chemical potential is $\mu_{\mathrm{L}}=e V / 2$. This implies that the occupation probability $p_{N}$ vanishes if $e V<2(N-1) U$, which is consistent with the occurrence of the steps shown in Fig. 3(b). In particular, for $e|V|<2 U$, only single occupation plays a role. Below we will use this fact for establishing an analytical treatment in the low-bias regime.

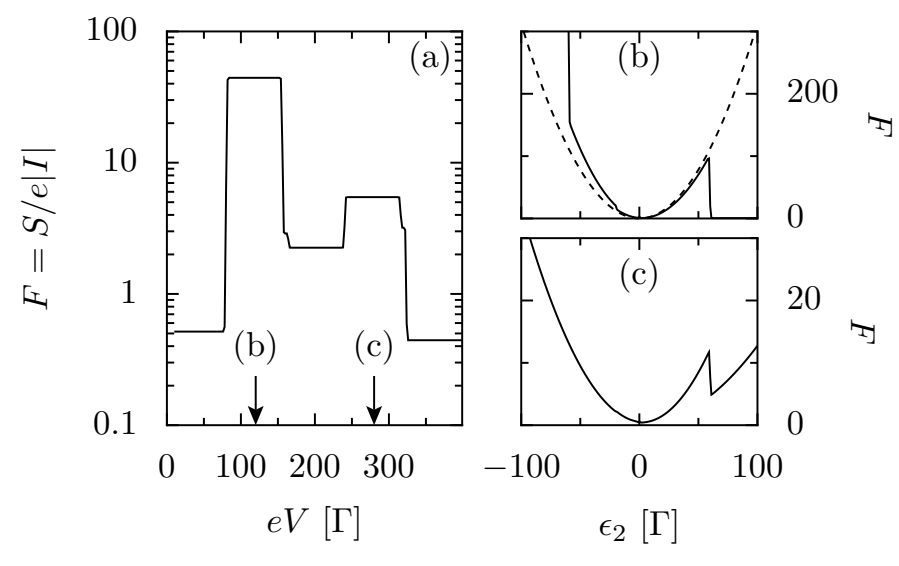

Figure 4: (a) Fano factor of the current shown in Fig. 31(a) for the interaction strength $U=80 \Gamma$. (b,c) Fano factor as a function of the detuning $\epsilon_{2}=e V_{\text {gate }}$ for the bias voltages (b) $V=120 \Gamma / e$ and (c) $V=280 \Gamma / e$. The dashed line in panel (b) represents the analytical result 27. valid in the one-electron regime.

\subsection{Shot noise and Fano factor}

So far we have seen that unless the bias voltage is extremely low, the current is always of the order $e \Gamma / \hbar$, i.e. changing the gate voltage or the bias voltage modifies the current typically by a factor of the order unity. Figure 4 demonstrates that the noise, characterized by the Fano factor $F=S / e|I|$, generally exhibits a more significant dependence on both the bias and the gate voltage.

In the three-electron regime, i.e. for $e V>4 U$, the Fano factor is of order unity and almost independent of $\epsilon_{2}$. This indicates that the transport process is Poissonian. In the two-electron regime, i.e. for $U<e V / 2<2 U$, the Fano factor [Fig. 4(c)] exhibits a quadratic dependence on $\epsilon_{2}$ as well, but the absolute values are now significantly smaller.

A rather pronounced dependence on the gate voltage $V_{\text {gate }}=\epsilon_{2} / e$ is found in that part of the single-electron regime in which the left chemical potential is so large that all single particle levels lie within the voltage window, i.e. for $\epsilon_{2}<e V / 2<U$; see Fig. 4(b). In particular, we observe a quadratic growth of the Fano factor, $F \propto \epsilon_{2}^{2}$, with highly super-Poissonian values. This already indicates electron bunching, where each bunch consists of roughly $F$ electrons [40].

\subsection{Full counting statistics}

A more complete picture can be drawn by considering the distribution function of the transported electrons or equivalently all cumulants, i.e., the full counting statistics. Here we restrict ourselves to the corresponding long-time limit which characterizes the low-frequency fluctuations. For avalanche-like transport, higher-order cumulants were computed [23, 25, 27, 28, 29] and recently also measured [41].

In a simplified picture, one may consider the electron avalanches as particles with charge $q=e F$ that are transported in an uncorrelated manner. For this Poisson pro- 


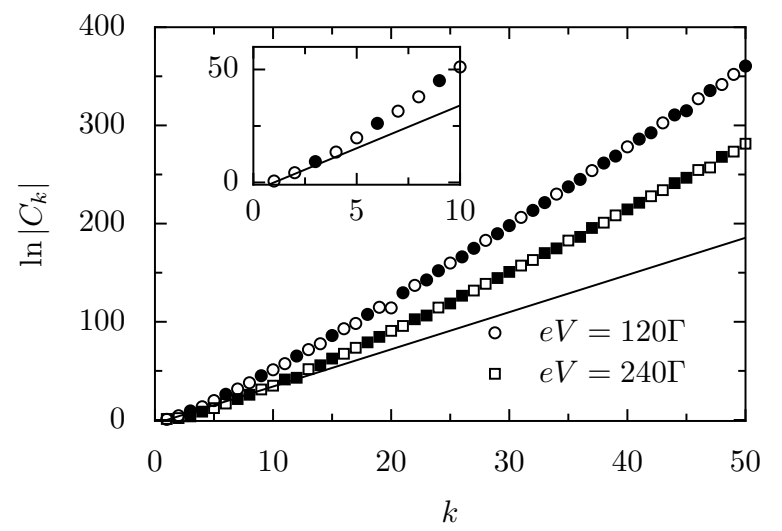

Figure 5: Full counting statistics of the current for $\epsilon_{2}=40 \Gamma$ and the bias voltages $V=120 \Gamma / e$ (one-electron regime) and $V=280 \Gamma / e$ (two-electron regime) expressed by the cumulants $C_{k}$. All other parameters are as in Fig. 4 Open symbols mark positive values while filled symbols correspond to negative values. The line marks the behavior for uncorrelated tunneling of charges $q=e F$ in the oneelectron regime (circles), cf. Eq. (20). Inset: Enlargement of the lower left corner.

cess, the cumulants (8) grow exponentially fast with their index according to the relation [42]

$$
C_{k}=\left(\frac{q}{e}\right)^{k-1} C_{1}=F^{k-1} C_{1}
$$

Closer theoretical investigations of avalanches with finite duration 25, 34], however, indicate that this picture deserves some refinement. In fact there it was found that the cumulants grow even faster. In avalanche diodes, by contrast, the opposite was measured, namely that the cumulants do not grow as fast [41]. But so far the underlying mechanism has not been revealed.

Figure 5 depicts the super exponential growth of the $C_{k}$ in both the one-electron regime and the two-electron regime. The solid line demonstrates that only the cumulants of very low order follow the behavior of the Poisson process underlying Eq. (20). Thus, we also in our case observe that already the third cumulant slightly exceeds the value $F^{2} C_{1}$, while higher-order cumulants even assume significantly larger absolute values. It is also interesting to notice that the sign of the cumulants changes periodically with the index $k$. This hints on the recently conjectured "universal cumulant oscillations" [43].

\section{Analytical solution in the one-electron regime}

If the bias voltage is so small that only one electron can reside on the triple dot, i.e. for $\mathrm{eV}<2 U$, all relevant eigenstates of $H_{\mathrm{TQD}}$ are the empty state $|0\rangle$ and the oneelectron states. To lowest order in $T / \epsilon_{2}$, the latter are given in the basis of the localized states $|j\rangle=c_{j}^{\dagger}|0\rangle$ by the expressions

$$
\begin{aligned}
& \left|\phi_{1}\right\rangle=\frac{1}{\sqrt{2}}(|1\rangle-|3\rangle), \\
& \left|\phi_{2}\right\rangle=\frac{1}{\sqrt{2+4\left(T / \epsilon_{2}\right)^{2}}}\left(|1\rangle-\frac{2 T}{\epsilon_{2}}|2\rangle+|3\rangle\right), \\
& \left|\phi_{3}\right\rangle=\frac{1}{\sqrt{1+2\left(T / \epsilon_{2}\right)^{2}}}\left(\frac{T}{\epsilon_{2}}|1\rangle+|2\rangle+\frac{T}{\epsilon_{2}}|3\rangle\right) .
\end{aligned}
$$

For symmetry reasons, each of these states couples with equal strength to the left and to the right lead, such that the corresponding incoherent transition rates from and to the leads are given by

$$
\Gamma_{\mathrm{L}, n}=\Gamma_{\mathrm{R}, n}=\Gamma\left|\left\langle\phi_{n} \mid 3\right\rangle\right|^{2} \equiv \Gamma_{n} .
$$

For the approximate eigenstates (21)-23) the coupling constants read $\Gamma_{1}=\Gamma_{2}=\Gamma / 2$ and $\Gamma_{3}=\Gamma T^{2} / \epsilon_{2}^{2}$.

If the dot-lead coupling $\Gamma$ is sufficiently small, one can neglect within a rotating-wave approximation off-diagonal elements of the reduced density operator [10], such that the system is well described by the occupation probabilities $\left(P_{0}, P_{1}, P_{2}, P_{3}\right)$ for the eigenstates $|0\rangle$ and $\left|\phi_{n}\right\rangle, n=1,2,3$. The corresponding Liouville operator is

$$
\mathcal{L}_{1 e}=\left(\begin{array}{cccc}
-\Gamma & \Gamma_{1} & \Gamma_{2} & \Gamma_{3} \\
\Gamma_{1} & -\Gamma_{1} & 0 & 0 \\
\Gamma_{2} & 0 & -\Gamma_{2} & 0 \\
\Gamma_{3} & 0 & 0 & -\Gamma_{3}
\end{array}\right),
$$

where the index " $1 e$ " refers to the restriction to single occupation. We have assumed that the chemical potentials are such that all three one-electron states lie within the voltage window. Moreover, we have employed the sum rule $\Gamma=\sum_{n=1}^{3} \Gamma_{n}$ which follows from the completeness relation for the eigenstates $\left|\phi_{n}\right\rangle$ in the one-electron subspace. The corresponding particle current operators are $\mathcal{J}^{-}=0$, while $\mathcal{J}^{+}$is obtained from $\mathcal{L}_{1 e}$ by keeping only the elements placed above the diagonal.

The stationary state of the Liouville operator can now be readily computed and reads $P_{0}=1 / 4=P_{n}$ for all $n$. Inserting this into the current formula (17), yields $I=$ $e \Gamma / 4 \hbar$. Interestingly enough, the current does not depend on the structure of the eigenstates, which is a consequence of the mentioned sum rule for the $\Gamma_{n}$.

For the Liouville operator (25), not only the stationary current, but also the zero-frequency noise (18) can be evaluated exactly. Starting from the latter expression, one obtains after some lines of straightforward calculation the result

$$
S=\frac{e^{2}}{32 \hbar} \sum_{n, n^{\prime}=1}^{3} \frac{\Gamma_{n}^{2}}{\Gamma_{n^{\prime}}}+\frac{e^{2}}{16 \hbar} \sum_{n=1}^{3} \frac{\Gamma_{1} \Gamma_{2} \Gamma_{3}}{\Gamma_{n}^{2}} .
$$

In the limit $\Gamma_{1,2} \ll \Gamma_{3}$, only terms with $\Gamma_{3}$ in the denominator are relevant, so that we obtain $S=\left(e^{2} / \hbar\right) \Gamma^{2} / 32 \Gamma_{3}$. With the above expressions for $\Gamma_{n}$, the corresponding Fano factor becomes

$$
F_{1 e}=\frac{\epsilon_{2}^{2}}{8 T^{2}} .
$$


It indeed exhibits the predicted parabolic dependence on the gate voltage $V_{\text {gate }}=\epsilon_{2} / e$. A quantitative comparison of both the current and the Fano factor with the numerical results depicted in Fig. 4(b) yields a satisfactory agreement in the range considered here. This implies that our oneelectron model indeed captures the essential features of the electron avalanches through the triple quantum dot. Moreover, it implies that the (average) size of the avalanches is $q=e \epsilon_{2}^{2} / 8 T^{2}$.

The proportionality of the Fano factor to $\left(\epsilon_{2} / T\right)^{2}$ suggests for the transport in the one-electron regime the following scenario: Assume that the off-resonant dot is initially unoccupied, while electrons are transported via dots 1 and 3. Then, according to standard perturbation theory, electrons in these dots may tunnel to dot 2 with a probability amplitude proportional to the tunnel matrix element divided by the energy difference, i.e. with a probability $p \propto\left(T / \epsilon_{2}\right)^{2}$. Thus after on average $1 / p$ electrons have been transported, an electron will tunnel to dot 2 and cause temporary Coulomb blockade which is resolved only when the electron tunnels further. Thus, the dynamics is given by periods with an open channel that on average terminate after an avalanche with $1 / p \propto(T / \epsilon)^{2}$ electrons has been transported. This explains the observed proportionality of the Fano factor $F \propto\left(\epsilon_{2} / T\right)^{2}$. This scenario also hints on why the cumulants deviate from conjecture (20): The average duration of an avalanche is as long as the waiting time between subsequent avalanches, while conjecture (20) is based on the assumption of a much shorter avalanche duration.

In the limit $\Gamma_{3} \ll \Gamma$, it is still possible to evaluate some cumulants of higher order analytically, although this becomes increasingly tedious. Nevertheless it is worth proceeding up to forth order for which we obtain

$$
\begin{aligned}
C_{1} & =\frac{\Gamma}{4 \hbar} \\
C_{2} & =\frac{\Gamma}{4 \hbar}\left(\frac{\Gamma}{8 \Gamma_{3}}\right), \\
C_{3} & =-\frac{3 \Gamma}{4 \hbar}\left(\frac{\Gamma}{8 \Gamma_{3}}\right)^{2}, \\
C_{4} & =\frac{3 \Gamma}{4 \hbar}\left(\frac{\Gamma}{8 \Gamma_{3}}\right)^{3} .
\end{aligned}
$$

Thus, the first two cumulants behave as expected for short avalanches with charge $q=e \Gamma / 8 \Gamma_{3}$. However, already the third and the forth cumulant deviate from the Poissonian value by a factor \pm 3 in compliance with our numerical observations in Sec. 3.3 .

\section{Conclusions}

The emergence of an interference pattern with good visibility usually requires the coherent superposition of two or more paths that are traversed with like probabilities. In an Aharonov-Bohm interferometer formed by quantum dots in ring configuration, a significant detuning of the dot that is not connected to any lead has the consequence that transport through one arm requires co-tunneling. Since this reduces the transmission probability of that path, the interference pattern as a function of a penetrating flux will fade away. We have demonstrated that, nevertheless, strongly detuned interferometers bear interesting effects manifest in the noise properties of the current. In particular, we have shown that strong electron bunching may occur. It turned out that this is most pronounced in the low bias regime in which Coulomb repulsion forbids the occupation of the triple quantum dot by more than one electron.

The prime quantity of interest in that context is the Fano factor for which we have predicted huge values: the relative noise strength may exceed that of a Poisson process by several orders of magnitude. This has led us to the conclusion that the current consists of avalanches with a finite duration. The physical reason for this is that electrons may become trapped in the off-resonant quantum dot, such that Coulomb blockade interrupts the transport until the trapped electron is released. The analysis of the higher-order charge fluctuations - the full-counting statistics - has revealed that the cumulants grow even super exponentially with their order.

For the computation of the full-counting statistics, we have employed the iterative scheme recently developed in Ref. [33]. This has been essential for the treatment of the three-dot problem, since the Hilbert space is already too large for a full analytical treatment. Nevertheless, in the most relevant regime of single occupation, we have performed the first iteration steps analytically, such that we have obtained expressions for the first four cumulants in the limit of strong detuning.

In conclusion, we have studied electron interferometers in a regime that so far has not attracted much attention, most likely due to the lack of pronounced interference effects. Precisely in this regime, however, the shot noise properties are most interesting and strong electron bunching occurs. Thus, quantum dots in ring configuration may serve not only for the observation of interference effects, but also for the creation of currents with widely tunable super-Poissonian fluctuations.

\section{Acknowledgements}

S.K. likes to thank Peter Hänggi for numerous inspiring discussions during many years of fruitful collaboration. We acknowledge helpful discussions with Christian Flindt. This work has been supported by the Spanish Ministry of Science and Innovation through project MAT2008-02626, a Ramón y Cajal fellowship (S.K.), and a FPI grant (F.D.).

\section{Appendix A. Rayleigh-Schrödinger perturbation theory}

In most textbooks on quantum mechanics, perturbation theory is formulated for a perturbation linear in the 
small parameter, while the augmented Liouvillian $\mathcal{L}_{\chi}$ contains arbitrarily high powers of $\chi$; see Eq. (10). In this appendix we derive a recursive scheme for the representation of an eigenvalue as a Taylor series in a perturbation parameter. Our calculation is inspired by Chap. 5 of Ref. [44], for an alternative derivation see Ref. [45]. Despite the fact that we formulate the problem in terms of a quantum mechanical energy eigenvalue problem, our derivation is not restricted to Hermitian operators.

We consider a "Hamiltonian" $H=H_{0}+V(\alpha)$ for which the perturbation $V(\alpha)$ is an analytical function of the perturbation parameter $\alpha$ and vanishes in the limit $\alpha \rightarrow 0$. Thus, it can be decomposed into the series

$$
V(\alpha)=\sum_{k=1}^{\infty} \alpha^{k} V_{k}
$$

Our goal is to find a series for the eigenvalue $E(\alpha)$ of $H$ that fulfills $\lim _{\alpha \rightarrow 0} E(\alpha)=E_{0}$, where $E_{0}$ is the eigenvalue of $H_{0}$ corresponding to a particular eigenvector $\left|\phi_{0}\right\rangle$, i.e., $H_{0}\left|\phi_{0}\right\rangle=E_{0}\left|\phi_{0}\right\rangle$. A central assumption is that both the eigenvalue and the corresponding eigenvector $|\phi(\alpha)\rangle$ can be decomposed into a series in $\alpha$ as well, such that we can employ the ansatz

$$
\begin{gathered}
E(\alpha)=E_{0}+\sum_{k=1}^{\infty} \alpha^{k} E_{k}, \\
|\phi(\alpha)\rangle=\left|\phi_{0}\right\rangle+\sum_{k=1}^{\infty} \alpha^{k}\left|\phi_{k}\right\rangle .
\end{gathered}
$$

For the present purpose, it is sufficient to consider only the case of non-degenerate $E_{0}$. Note that for non-Hermitian $H_{0},\left\langle\phi_{0}\right|$ is generally not the Hermitian adjoint of $\left|\phi_{0}\right\rangle$, but rather the corresponding left eigenvector, i.e., the solution of $\left\langle\phi_{0}\right| H_{0}=E_{0}\left\langle\phi_{0}\right|$. We choose the normalization such that $\left\langle\phi_{0} \mid \phi_{0}\right\rangle=1$.

We start by writing the eigenvalue equation $H|\phi(\alpha)\rangle=$ $E(\alpha)|\phi(\alpha)\rangle$ in the form

$$
\left(H_{0}-E_{0}\right)|\phi(\alpha)\rangle=\left\{E(\alpha)-E_{0}-V(\alpha)\right\}|\phi(\alpha)\rangle,
$$

which is convenient for the derivation of a formal solution and subsequent iteration. Since $\left(H_{0}-E_{0}\right)\left|\phi_{0}\right\rangle=0$, Eq. (A.4) defines $|\phi(\alpha)\rangle$ only up to a component proportional to $\left|\phi_{0}\right\rangle$. Moreover, it implies that the inverse of $H_{0}-E_{0}$ does not exist. It is however possible to define the pseudo-inverse $Q\left(H_{0}-E_{0}\right)^{-1} Q$ [or in short: $Q /\left(H_{0}-E_{0}\right)$ ], where $Q$ is the projector on the subspace spanned by the eigenvectors of $H_{0}$ with non-zero eigenvalue. In the present case of non-degenerate $E_{0}$, it reads $Q=\mathbf{1}-\left|\phi_{0}\right\rangle\left\langle\phi_{0}\right|$.

The pseudo-inverse allows one to cast Eq. (A.4) into the form

$$
|\phi(\alpha)\rangle=\left|\phi_{0}\right\rangle+\frac{Q}{H_{0}-E_{0}}\left\{E(\alpha)-E_{0}-V(\alpha)\right\}|\phi(\alpha)\rangle .
$$

The first term on the right-hand side can be multiplied by any factor without violating Eq. (A.4). We have chosen it such that $\lim _{\alpha \rightarrow 0}|\phi(\alpha)\rangle=\left|\phi_{0}\right\rangle$. Moreover, since $\left\langle\phi_{0}\right| Q=0$, the relation $\left\langle\phi_{0} \mid \phi(\alpha)\right\rangle=\left\langle\phi_{0} \mid \phi_{0}\right\rangle=1$ holds. An important feature of Eq. (A.5) is that the second term on the right-hand side is of first order in $\alpha$. Therefore, it can be solved iteratively in the following way.

Multiplying Eq. (A.4) by $\left\langle\phi_{0}\right|$, we find that the lefthand-side vanishes owing to $\left\langle\phi_{0}\right| H_{0}=E_{0}\left\langle\phi_{0}\right|$. Thus we obtain $E(\alpha)-E_{0}=\left\langle\phi_{0}|V(\alpha)| \phi(\alpha)\right\rangle$. We then insert for $E(\alpha), V(\alpha)$, and $|\phi(\alpha)\rangle$ the series A.1 A.3 and compare coefficients to obtain the $k$ th-order energy shift

$$
E_{k}=\sum_{k^{\prime}=1}^{k}\left\langle\phi_{0}\left|V_{k^{\prime}}\right| \phi_{k-k^{\prime}}\right\rangle
$$

It depends on the still unknown correction of the eigenvector, $\left|\phi_{k-k^{\prime}}\right\rangle$, which we determine from Eq. (A.5). We again insert Eqs. A.1 A.3 and compare coefficients to find

$$
\left|\phi_{k}\right\rangle=\frac{Q}{H_{0}-E_{0}}\left\{\sum_{k^{\prime}=1}^{k-1} E_{k^{\prime}}\left|\phi_{k-k^{\prime}}\right\rangle-\sum_{k^{\prime}=1}^{k} V_{k^{\prime}}\left|\phi_{k-k^{\prime}}\right\rangle\right\} .
$$

Equations (A.6) and (A.7) allow the recursive computation of the series (A.2) for the eigenvalue shift $E(\alpha)$.

\section{References}

[1] R. P. Feynman, Eng. Sci. 23 (1960) 22, lecture given at the APS meeting 1959, see http://www.its.caltech.edu/ feynman/plenty.html.

[2] J. C. Ellenbogen, J. C. Love, Proc. IEEE 88 (2000) 386.

[3] P. Hänggi, M. Ratner, S. Yaliraki, Chem. Phys. 281 (2002) 111502.

[4] G. Cuniberti, G. Fagas, K. Richter (Eds.), Molecular Electronics, Lecture Notes in Physics, Springer, Berlin, 2005.

[5] F. Grossmann, T. Dittrich, P. Jung, P. Hänggi, Phys. Rev. Lett. 67 (1991) 516.

[6] C. E. Creffield, G. Platero, Phys. Rev. B 65 (2002) 113304.

[7] J. Lehmann, S. Camalet, S. Kohler, P. Hänggi, Chem. Phys. Lett. 368 (2003) 282.

[8] S. Camalet, J. Lehmann, S. Kohler, P. Hänggi, Phys. Rev. Lett. 90 (2003) 210602.

[9] G. Platero, R. Aguado, Phys. Rep. 395 (2004) 1.

[10] S. Kohler, J. Lehmann, P. Hänggi, Phys. Rep. 406 (2005) 379.

[11] N. C. van der Vaart, S. F. Godijn, Y. V. Nazarov, C. J. P. M. Harmans, J. E. Mooij, Phys. Rev. Lett. 74 (1995) 4702.

[12] R. H. Blick, R. J. Haug, J. Weis, D. Pfannkuche, K. von Klitzing, K. Eberl, Phys. Rev. B 53 (1996) 7899.

[13] W. G. van der Wiel, S. De Franceschi, J. M. Elzerman, T. Fujisawa, S. Tarucha, L. P. Kouwenhoven, Rev. Mod. Phys. 75 (2003) 1.

[14] D. Schröer, A. D. Greentree, L. Gaudreau, K. Eberl, L. C. L. Hollenberg, J. P. Kotthaus, S. Ludwig, Phys. Rev. B 76 (2007) 075306.

[15] L. Gaudreau, S. A. Studenikin, A. S. Sachrajda, P. Zawadzki, A. Kam, J. Lapointe, M. Korkusinski, P. Hawrylak, Phys. Rev. Lett. 97 (2006) 036807.

[16] M. C. Rogge, R. J. Haug, Phys. Rev. B 77 (2008) 193306.

[17] S. Gustavsson, M. Studer, R. Leturcq, T. Ihn, K. Ensslin, D. C. Driscoll, A. C. Gossard, Phys. Rev. Lett. 99 (2007) 206804.

[18] B. Michaelis, C. Emary, C. W. J. Beenakker, Europhys. Lett. 73 (2006) 677.

[19] C. Pöltl, C. Emary, T. Brandes, Phys. Rev. B 80 (2009) 115313.

[20] R. Sanchez, E. Cota, R. Aguado, G. Platero, Phys. Rev. B 74 (2006) 035326. 
[21] M. Busl, R. Sánchez, G. Platero, Phys. Rev. B 81 (2010) 121306(R).

[22] R. Sánchez, S. Kohler, G. Platero, New J. Phys. 10 (2008) 115013.

[23] C. Flindt, T. Novotný, A.-P. Jauho, Phys. Rev. B 70 (2004) 205334.

[24] J. Koch, F. von Oppen, Phys. Rev. Lett. 94 (2005) 206804.

[25] W. Belzig, Phys. Rev. B 71 (2005) 161301(R).

[26] E. Vernek, C. A. Büsser, G. B. Martins, E. V. Anda, N. Sandler, S. E. Ulloa, Phys. Rev. B 80 (2009) 035119

[27] D. Urban, J. König, R. Fazio, Phys. Rev. B 78 (2008) 075318.

[28] D. Urban, J. König, Phys. Rev. B 79 (2009) 165319.

[29] F. Li, H. Jiao, J. Luo, X.-Q. Li, S. Gurvitz, Physica E 41 (2009) 1707.

[30] G. Schaller, G. Kießlich, T. Brandes, Phys. Rev. B 80 (2009) 245107.

[31] G. B. Lesovik, L. S. Levitov, Phys. Rev. Lett. 72 (1994) 538.

[32] D. A. Bagrets, Yu. V. Nazarov, Phys. Rev. B 67 (2003) 085316.

[33] C. Flindt, T. Novotný, A. Braggio, M. Sassetti, A.-P. Jauho, Phys. Rev. Lett. 100 (2008) 150601.

[34] C. Flindt, T. Novotný, A. Braggio, A.-P. Jauho, arXiv:1002.4506 [cond-mat].

[35] K. Blum, Density Matrix Theory and Applications, 2nd Edition, Springer, New York, 1996.

[36] U. Fano, Phys. Rev. 72 (1947) 26.

37] F. J. Kaiser, S. Kohler, Ann. Phys. (Leipzig) 16 (2007) 702

[38] C. Emary, Phys. Rev. B 76 (2007) 245319.

[39] E. Arimondo, Prog. Opt. 35 (1996) 259.

[40] L. S. Levitov, M. Reznikov, Phys. Rev. B 70 (2004) 115305.

[41] J. Gabelli, B. Reulet, Phys. Rev. B 80 (2009) 161203(R).

[42] N. G. van Kampen, Stochastic processes in physics and chemistry, North-Holland, Amsterdam, 1992.

[43] C. Flindt, C. Fricke, F. Hohls, T. Novotný, K. Netočný, T. Brandes, R. J. Haug, Proc. Natl. Acad. Sci. USA 106 (2009) 10116.

[44] J. J. Sakurai, Modern Quantum Mechanics, 2nd Edition, Addison-Wesley, Reading, 1995.

[45] M. Baiesi, C. Maes, K. Netočný, J. Stat. Phys. 135 (2009) 57. 